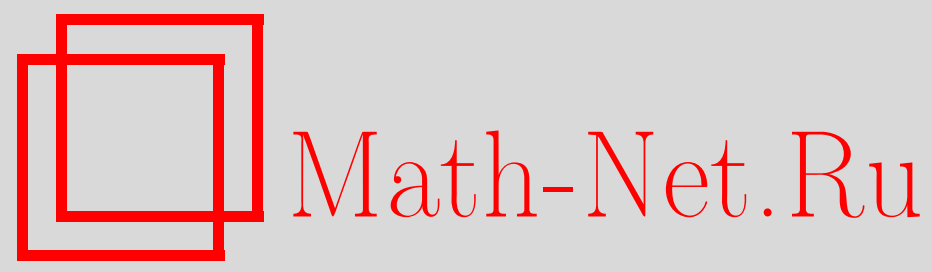

Е. М. Вечтомов, Решетка подалгебр колец непрерывных функций и хьюиттовские пространства, Матем. заметки, 1997, том 62, выпуск 5, 687-693

DOI: https://doi.org/10.4213/mzm1655

Использование Общероссийского математического портала Math-Net.Ru подразумевает, что вы прочитали и согласны с пользовательским соглашением http://www.mathnet.ru/rus/agreement

Параметры загрузки:

IP: 34.239 .49 .27

26 апреля 2023 г., 18:01:03

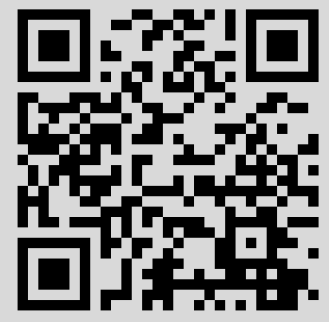




\section{РЕШЕТКА ПОДАЛГЕБР КОЛЕЦ НЕПРЕРЫВНЫХ ФУНКЦИЙ И ХЬЮИТТОВСКИЕ ПРОСТРАНСТВА}

\section{Е. М. Вечтомов}

Рассматривается решетка $A(X)$ всевозможных подалгебр кольцавсех непрерывных $\mathbb{R}$-значных функций, определенных на $\mathbb{R}$-отделимом пространстве $X$. Топологическое пространство назьвается хьюиттовским, если оно гомеоморфно замкнутому подпространству некоторой тихоновской степени числовой прямой $\mathbb{R}$. Основным результатом работы является доказательство определяемости любого хьюиттовского пространства $X$ решеткой $A(X)$. Применяется оригинальная техника минимальных и максимальных подалгебр. Показано, что модулярность решетки $A(X)$ равносильна тому, что $X$ содержит не более двух точек.

Библиография: 3 названия.

Введение. Целью статьи является доказательство определяемости любого хьюиттовского пространства $X$ решеткой всех подалгебр кольца непрерьвных функций на $X$. Применяется оригинальная техника минимальных и максимальных подалгебр. Исследуются также некоторые алгебраические свойства решетки подалгебр кольца непрерьвных функций.

Пусть $X$ - топологическое пространство и $C(X)$ - кольцо всех непрерьвных $\mathbb{R}-$-зачных отображений, заданных на $X$, с поточечно определенньми операциями. Как обычно, $\mathbb{R}$ - это топологическоеполе действительных чисел. Обозначим через $A(X)$ решетку всех $\mathbb{R}$-подалгебр кольца $C(X)$ относительно отношения включения ' $\subseteq$ '. Решетка $A(X)$ является полной с наименьшим элементом 0 и наибольшим элементом $C(X)$. Минимальные (ненулевые) подалгебры в $C(X)$ - это атомы решетки $A(X)$, а максимальные (собственные) подалгебры - ее коатомы.

Хаусдорфово пространство $X$ назьвается тихоновским, если для любого замкнутого множества $V \subseteq X$ и любой точки $x \in X \backslash V$ найдется функция $f \in C(X)$ такая, что $f(V)=\{0\}$ и $f(x)=1$. Тихоновское пространство $X$ называется хьюиттовским (или $Q$-пространством, вещественно полным, функционально замкнутым), если все $\mathbb{R}$-идеалы кольца $C(X)$ являются фиксированньпи максимальньми идеалами. Напомним, что идеал $M$ в $C(X)$ называется $\mathbb{R}$-идеалом, если факторкольцо $C(X) / M$ изоморфно полю $\mathbb{R}$. Идеалы вида $M_{x}=\{f \in C(X): f(x)=0\}, x \in X$, называются фиксированнымми максимальными идеалами. Хорошо известны следующие характеризации (см., например, [1]): топологическое пространство является тихоновским (хьюиттовским) тогда и только тогда, когда оно гомеоморфно подпространству (замкнутому подпространству) некоторой тихоновской степени пространства $\mathbb{R}$. Топологическое пространство $X$ назьвается $\mathbb{R}$-отделимым, если для любых двух различных его точек $x$ и $y$ существует $f \in C(X)$, для которой $f(x) \neq f(y)$. Нуль-множеством на $X$ назьвается множество 
вида $Z(f)=\{x \in X: f(x)=0\}, f \in C(X)$. Множество coz $f=X \backslash Z(f)$ при $f \in C(X)$ назьвается конуль-множеством.

Говорят, что хъюиттовское пространство $X$ определяется решеткой $A(X)$, если для любого хьюиттовского пространства $Y$ изоморфность решеток $A(Y) \cong A(X)$ влечет гомеоморфность пространств $Y \approx X$. Проблема определяемости хьюиттовских пространств $X$ решетками $A(X)$ ставилась автором на Тираспольской школе по топологической алгебре в августе 1988 года и на Общемосковском топологическом семинаре имени П. С. Александрова в мае 1994 года. Определяемость произвольного хьюиттовского пространства $X$ кольцом $C(X)$ доказана Хьюиттом еще в 1948 году. Теории определяемости топологических пространств посвящен обзор [2].

Следующие две теоремы являются основньми в работе.

Теорема 1. Всякое хъюиттовское пространство X определяется решеткой $A(X)$.

ТЕОРема 2. Для любых двух топологических пространств $X$ и $Y$ әквивалентны условия:

1) решетки $A(X)$ и $A(Y)$ изоморфны;

2) кольиа $C(X)$ и $C(Y)$ изоморфны.

Теорема 2 вытекает из теоремы 1. Действительно, поскольку любой изоморфизм колец $C(X)$ и $C(Y)$ является их изоморфизмом как $\mathbb{R}$-алгебр $[1,1 \mathrm{~J}]$, то 2) влечет 1$)$. Известно также [1, теоремы 3.9 и 8.7], что для произвольного топологического пространства $Z$ существуют тихоновское пространство $\tau Z$ и хьюиттовское пространство $\nu \tau Z$, для которых кольца $C(Z), C(\tau Z)$ и $C(\nu \tau Z)$ канонически изоморфны. Пусть теперь $A(X) \cong A(Y)$. Тогда $A(\nu \tau X) \cong A(\nu \tau Y)$. По теореме $1 \nu \tau X \approx \nu \tau Y$. Стало быть, $C(X) \cong C(\nu \tau X) \cong C(\nu \tau Y) \cong C(Y)$. Значит, и условие 1) влечет условие 2$)$.

Отметим, что необходимые теоретико-решеточные понятия можно найти в книге [3].

1. Минимальные и максимальные подалгебры. Пока на топологическое пространство $X$ не накладывается никаких ограничений. Подалгебры $\mathbb{R}$-алгебры $C(X)$ будем назьвать также подалгебрами на $X$. Заметим, что все идеалы кольца $C(X)$ являются подалгебрами на $X$. Решеточными операциями в $A(X)$ служат $A \cap B$ и $A \vee B=$ $A+B+A B$, где $A B=\left\{\sum a_{i} b_{i}: a_{i} \in A, b_{i} \in B\right\}$.

Для $f \in C(X)$ пусть $[f] \in A(X)$ - подалгебра, порожденная $f$. Конечно, $[f]$ представляет собой подалгебру на $X$ всех многочленов от $f$ с действительньми коэффициентами и без свободных членов.

Минимальные подалгебры на $X$ легко описьваются.

Лемма 1. Подалгебра $A \in A(X)$ минимальна тогда и только тогда, когда $A=e \mathbb{R}$ для некоторого ненулевого идемпотента е из $C(X)$.

ДоказАтельство. Очевидно, что подалгебры $e \mathbb{R}, e \neq 0$ и $e^{2}=e \in C(X)$, минимальны. Предположим, что $A$ - минимальная подалгебра на $X$. Возьмем ненулевую функцию $f \in A$ и рассмотрим подалгебру $\left[f^{2}\right]$. Тогда $A=\left[f^{2}\right]$, и $f=f^{2} g$ для подходящего многочлена $g$ от $f$ с действительными коэффициентами. Положив $e=f g \in A$, имеем $Z(e)=Z(f)$ и $e=1$ на $\operatorname{coz} f$. Следовательно, $e^{2}=e$ и $A=e \mathbb{R}$.

Лемма 2. Для любых различных минимальных подалгебр А и $В$ на $X$ подалгебра $A \vee B$ содержит ровно 3 или 7 минимальных подалгебр из $A(X)$. 
ДокАЗАтЕльство. Пусть $A \neq B$ - минимальные подалгебры на $X$. По лемме 1 $A=e \mathbb{R}$ и $B=f \mathbb{R}$ для ненулевых идемпотентов $e \neq f$ из $C(X)$. Если $Z(e) \subset Z(f)$, $Z(f) \subset Z(e)$ или $e f=0$, то $e \mathbb{R} \vee f \mathbb{R}$ содержит в точности 3 минимальные подалгебры: $e \mathbb{R}, f \mathbb{R}$ и $(e+f-2 e f) \mathbb{R}$. В противном случае, т.е. когда coz $е$ и coz $f$ находятся в общем положении, подалгебра $e \mathbb{R} \vee f \mathbb{R}$ содержит 7 минимальных подалгебр на $X$, а именно: $e \mathbb{R}, f \mathbb{R}, e f \mathbb{R},(e-e f) \mathbb{R},(f-e f) \mathbb{R},(e+f-2 e f) \mathbb{R}$ и $(e+f-e f) \mathbb{R}$.

При $x \neq y$ из $X$ обозначим через $A_{x, y}$ подалгебру всех функций $f \in C(X)$, для которых $f(x)=f(y)$. Следующее утверждение очевидно.

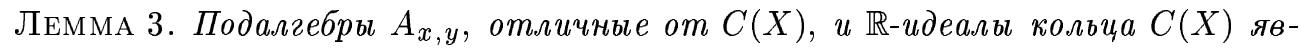
ляются максимальными подалгебрами на $X$.

Лемма 4. Для собственной подалгебры $A$ на $X$

$$
A \vee \mathbb{R}=C(X) \Longleftrightarrow A \text { есть } \mathbb{R}-\text { идеал в } C(X) \text {. }
$$

ДокаЗАТЕЛЬСТво. Достаточнопроверить импликацию ' $\Longrightarrow$ '. Пусть $A \vee \mathbb{R}=C(X)$. Тогда $A+\mathbb{R}=C(X)$. Если $f \in A$ и $g \in C(X)$, то $g=h+r$ для некоторых $h \in A$ и $r \in \mathbb{R}$ и $f g=f h+f r \in A$. Значит, $A$ - идеал кольца $C(X)$ и $C(X) / A \cong \mathbb{R}$.

ПРЕДЛОЖЕНИЕ 1. Пусть $X-$-ъюиттовское пространство, $M$ - максимальная подалгебра на $X, e \mathbb{R}$ - минимальная подалгебра на $X$, по крайней мере одно из множеств coz е или $Z\left(\right.$ е) компактно $и M \vee e \mathbb{R}=C(X)$. Тогда $M=M_{x}$ для некоторой точки $x \in X$, если $1 \notin M, u M=A_{x, y}$ для подходящих точек $x \in \operatorname{coz} e$ u $y \in Z(e)$, если $1 \in M$.

ДоКАЗАТЕЛЬСТво. По лемме $4 M$ имеет вид $M_{x}$ при $1 \notin M$.

Пусть $1 \in M$, т.е. $\mathbb{R} \subseteq M$. Тогда $M+e M=M+(1-e) M=C(X)$. Значит,

$$
\left.M\right|_{\operatorname{coz} e}=C(\operatorname{coz} e) \quad \text { и }\left.\quad M\right|_{Z(e)}=C(Z(e)) .
$$

Можно считать, что конуль-множество coz $e$ компактно; иначе вместо идемпотента $e$ надо взять идемпотент $1-e$. Допустим, что имеет место следующее свойство:

$$
(\forall x \in \operatorname{coz} e)(\exists f, g \in M)(f=g \text { на } Z(e) \text { и } f(x) \neq g(x)),
$$

из которого вытекает свойство

$$
(\forall x \in \operatorname{coz} e)(\exists h \in M)(h(Z(e))=\{0\} \text { и } h(x)=1) .
$$

Тогда в силу компактности coz $е$ очевидно существование функции $f \in M$ такой, что $Z(f)=Z(e)$ и $\operatorname{coz} f=\operatorname{coz} e$. Так как вьполнено $\left.M\right|_{\operatorname{coz} e}=C(\operatorname{coz} e)$, в $M$ найдется функция $g$, равная $f^{-1}$ на coz $e$. Но тогда получили бы $e=f g \in M$, что невозможно.

Следовательно, наше допущение неверно. Стало быть, существует точка $x \in \operatorname{coz} e$ такая, что

$$
(\forall f, g \in M)\left(\left.f\right|_{Z(e)}=\left.g\right|_{Z(e)} \Longrightarrow f(x)=g(x)\right) .
$$

Рассмотрим гомоморфизм $\alpha: C(Z(e)) \rightarrow \mathbb{R}, \alpha(\varphi)=f(x)$, где $f \in C(X)$ - единственное продолжение функции $\varphi \in C(Z(e))$, лежащее в $M$. Его ядро $\operatorname{Ker} \alpha$ есть $\mathbb{R}$-идеал кольца $C(Z(e))$. Пространство $Z(e)$ является хьюиттовским вместе с $X$. Поэтому Ker $\alpha=$ $M_{y} \subseteq C(Z(e))$ для некоторой (единственной) точки $y \in Z(e)$, и $\alpha$ есть вычисление в точке $y$. Теперь, если $f \in M$, то $f(x)=\alpha\left(\left.f\right|_{Z(e)}\right)=f(y)$. Значит, $M \subseteq A_{x, y}$, откуда $M=A_{x, y}$. Предложение доказано.

Если $p \in X-$ изолированная точка, т.е. множество $\{p\}$ открыто-замкнуто, то обозначим через $e_{p}$ идемпотент из $C(X)$, для которого $Z\left(e_{p}\right)=X \backslash\{p\}$. 
СЛЕДСТВИЕ 1. Пусть $p$ - изолированная точка хьюиттовского пространства $X, A \in A(X)$ и $A \vee e_{p} \mathbb{R}=C(X)$. Тогда $A=M_{p}$ или $A=A_{p, y}$ для некоторой точки $y \in X \backslash\{p\}$.

В самом деле, если $A \neq M_{p}$, то, как и в предложении 1 , получаем $A \subseteq A_{p, y}$ при $y \in X \backslash\{p\}$. А поскольку $A+e_{p} \mathbb{R}=A \vee e_{p} \mathbb{R}=C(X)$, то $A=A_{p, y}$.

СлЕДСТВИЕ 2. Пусть $X-$ нульмерный компакт и $M-$ максимальная подалгебра на $X$. Тогда $M \vee A=C(X)$ для некоторой минимальной подалгебры $A$ на $X \boldsymbol{в}$ том и только том случае, когда $M=M_{x}(x \in X)$ или $M=A_{x, y}(x \neq y$ из $X)$.

Напомним, что компакт - это компактное хаусдорфово пространство, а нульмерные пространства - это топологические пространства, обладающие базой из открыто-замкнутых множеств.

2. Отмеченные подалгебры. Трехэлементноемножество $\{e \mathbb{R}, f \mathbb{R}, g \mathbb{R}\}$ минимальных подалгебр на $X$ назовем простой тройкой в решетке $A(X)$, если $e \mathbb{R} \vee f \mathbb{R} \vee g \mathbb{R}$ содержит только 3 эти минимальные подалгебры; в силу леммы 2 это означает вьполнение одного из равенств: $e=f+g$ (при $f g=0), f=e+g$ или $g=e+f$.

В этом пункте через $M(X)$ будем обозначать множество всевозможных максимальных подалгебр на $X$. Для $Z \subseteq X$ положим $M_{Z}=\{h \in C(X): Z \subseteq Z(h)\}$.

Элемент $e \mathbb{R}$ простой тройки $\{e \mathbb{R}, f \mathbb{R}, g \mathbb{R}\}$ назовем отмеченным в этой тройке, если

$$
\begin{aligned}
& (\cap\{M \in M(X): M \vee e \mathbb{R}=C(X) \text { и } f \mathbb{R} \subseteq M\}) \\
& \quad \vee(\cap\{M \in M(X): M \vee e \mathbb{R}=C(X) \text { и } g \mathbb{R} \subseteq M\})=C(X) .
\end{aligned}
$$

Наконец, минимальную подалгебру на $X$ назовем отмеченной подалгеброй, если она является отмеченным элементом некоторой простой тройки в $A(X)$.

Подчеркнем, что понятия простой тройки, отмеченного элемента простой тройки в решетке $A(X)$ и отмеченной подалгебры на $X$ являются чисто решеточными.

В леммах 5 и 6 пространство $X$ предполагается хьюиттовским.

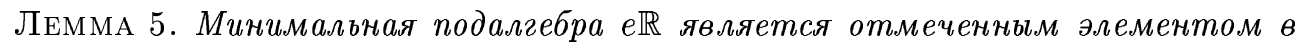
данной простой тройке $\{e \mathbb{R}, f \mathbb{R}, g \mathbb{R}\}$ решетки $A(X)$ тогда и только тогда, когда $e=1$ или $е=e_{p}$ для некоторой изолированной точки $p \in X$.

ДОКАЗАТЕЛЬСТВО. Пусть

$$
\begin{aligned}
& A=\cap\{M \in M(X): M \vee e \mathbb{R}=C(X) \text { и } M \ni f\}, \\
& B=\cap\{M \in M(X): M \vee e \mathbb{R}=C(X) \text { и } M \ni g\} .
\end{aligned}
$$

Проверим сначала отмеченность $e \mathbb{R}$ в случаях $e=1$ и $e=e_{p}$. Пусть $e=1$. Тогда $g=1-f$. По лемме 4

$$
A=\cap\left\{M_{x}: x \in Z(f)\right\}=M_{Z(f)}, \quad B=\cap\left\{M_{x}: x \in Z(g)\right\}=M_{\operatorname{coz} f} .
$$

Откуда $A \vee B=M_{Z(f)}+M_{\operatorname{coz} f}=C(X)$.

Пусть $e=e_{p}$ и, скажем, $g=e_{p}+f$. По следствию 1

$$
A=M_{p} \cap\left(\cap\left\{A_{p, x}: x \in Z(f)\right\}\right)=M_{Z(f)}, \quad B=\cap\left\{A_{p, x}: x \in \operatorname{coz} g\right\}=M_{\text {coz } g}+\mathbb{R} .
$$


Значит, $A \vee B=M_{Z(f)}+M_{\mathrm{coz} g}+\mathbb{R}=M_{p}+\mathbb{R}=C(X)$.

Покажем, что в остальных случаях $e \mathbb{R}$ не является отмеченным элементом простой тройки $\{e \mathbb{R}, f \mathbb{R}, g \mathbb{R}\}$. Это случаи: $e=f+g \neq 1$ и $g=e+f$ при $|\operatorname{coz} e| \geqslant 2$.

Пусть сначала $e=f+g \neq 1$. Тогда по лемме $3 A \subseteq \cap\left\{M \in M(X): M=M_{x}\right.$ при $x \in \operatorname{coz} g$ или $M=A_{y, z}$ при $\left.y \in \operatorname{coz} g, z \in Z(e)\right\}=M_{Z(f)}$. Аналогично, $B \subseteq M_{Z(g)}$. Поэтому

$$
A \vee B \subseteq M_{Z(f)}+M_{Z(g)}=M_{Z(e)} \neq C(X) .
$$

Наконец, пусть $g=e+f$ и $|\operatorname{coz} e| \geqslant 2$. Тогда

$$
\begin{gathered}
A \subseteq\left(\cap\left\{M_{x}: x \in \operatorname{coz} e\right\}\right) \cap\left(\cap\left\{A_{y, z}: y \in \operatorname{coz} e, z \in Z(g)\right\}\right)=M_{Z(f)}, \\
B \subseteq \cap\left\{A_{x, y}: x \in \operatorname{coz} e, y \in \operatorname{coz} f\right\}=M_{\operatorname{coz} g}+\mathbb{R} .
\end{gathered}
$$

Все функции из $A \vee B$ являются константами на множестве coz $e$, содержащем по крайней мере две точки. Значит, $A \vee B \neq C(X)$. Лемма доказана.

ПрЕДЛОЖЕНИЕ 2. Для всякого $\mathbb{R}$-отделимого пространства $X$ справедливы следуюшие утверждения:

а) әквивалентны условия:

$X$ связно;

на $X$ существует единственная минимальная подалгебра $\mathbb{R} ;$

на $X$ нет отмеченных подалгебр;

б) если $X$ несвязно, то отмеченными подалгебрами на $X$ служат в точности подалгебры $\mathbb{R}$ и е $e_{p} \mathbb{R}$ для изолированных точек $p$ пространства $X ;$

в) если $X$ несвязно, то оно имеет хотя бъ одну изолированную точку тогда и только тогда, когда на $X$ существует более одной отмеченной подалгебры;

г) существование в решетке $A(X)$ простой тройки, все әлементы которой отмеченные, равносильно тому, что $X$ - несвязное двоеточие.

ДокаЗАТЕЛЬСтво. Предложение 2 сразу следует из леммы 5 . Только надо учесть, что операции тихоновизации $\tau$ и хьюиттовского расширения $\nu$, о которых говорилось во введении, последовательно примененные к $\mathbb{R}$-отделимому пространству, сохраняют его связность и изолированные точки (в обе стороны).

Лемма 6. Пусть $\left\{\mathbb{R}, e_{p} \mathbb{R},\left(1-e_{p}\right) \mathbb{R}\right\}$ - простая тройка в $A(X)$ с отмеченными әлементами $e_{p} \mathbb{R} u \mathbb{R} . \quad$ Тогда $\left(1-e_{p}\right) \mathbb{R} \subseteq M_{p}$ и $M_{p}$ - единственный әлемент решетки $A(X)$, являющийся дополнением $u \mathbb{R}, u e_{p} \mathbb{R}$.

Лемма 6 вытекает из леммы 4 и следствия 1.

3. Доказательство теоремы 1. Пусть для произвольных хьюиттовских пространств $X$ и $Y$ дан изоморфизм $\alpha$ решетки $A(X)$ на решетку $A(Y)$. Рассмотрим $\alpha(\mathbb{R})$. На основании утверждений а) и б) предложения 2 имеем $\alpha(\mathbb{R})=e \mathbb{R}$, где $e=1$ или $e=e_{q}$ для некоторой изолированной точки $q \in Y$. Разберем эти случаи.

Первьй случай: $e=1$. По лемме 4 дополнениями $\mathbb{R}$ в решетке $A(X)($ в $A(Y)$ ) являются в точности фиксированные максимальные идеалы кольца $C(X)(C(Y))$. Поэтому изоморфизмы $\alpha$ и $\alpha^{-1}$ сохраняют фиксированные максимальные идеалы. Полагая для любых $x \in X$ и $y \in Y$

$$
\varphi(x)=y \Longleftrightarrow \alpha\left(M_{x}\right)=M_{y},
$$


получаем биекцию $\varphi$ пространства $X$ на пространство $Y$.

Покажем, что $\varphi$ - гомеоморфизм. Для этой цели возьмем произвольную функцию $f \in C(X)$ и подалгебру $[f] \in A(X)$. Подалгебра $\alpha([f]) \in A(Y)$ порождена некоторым конечным числом функций $g_{1}, \ldots, g_{n} \in C(Y)$. Действительно, конечная порожденность произвольной подалгебры $A \in A(Z)$ является решеточным свойством: $A$ - компактньй элемент решетки $A(Z)[3$, c. 243]. Имеем, далее,

$$
\begin{aligned}
\varphi(Z(f)) & =\varphi\left(\left\{x \in X:[f] \subseteq M_{x}\right\}\right)=\left\{y \in Y: \alpha([f]) \subseteq M_{y}\right\} \\
& =\bigcap_{i=1}^{n}\left\{y \in Y: g_{i} \in M_{y}\right\}=\bigcap_{i=1}^{n} Z\left(g_{i}\right)=Z\left(\sum_{i=1}^{n} g_{i}^{2}\right) .
\end{aligned}
$$

Итак, $\varphi$ сохраняет нуль-множества. Конечно, $\varphi^{-1}$ также сохраняет нуль-множества. Поскольку нуль-множества образуют базу замкнутых множеств в любом тихоновском пространстве, то биекция $\varphi$ осуществляет гомеоморфность пространств $X$ и $Y$.

Второй случай: $e=e_{q}$. В силу утверждения б) предложения $2 \alpha^{-1}(\mathbb{R})=e_{p} \mathbb{R}$ для подходящей изолированной точки $p \in X$. Простая тройка $\left\{\mathbb{R}, e_{p} \mathbb{R},\left(1-e_{p}\right) \mathbb{R}\right\}$ в $A(X)$ переводится изоморфизмом $\alpha$ в простую тройку $\left\{e_{q} \mathbb{R}, \mathbb{R},\left(1-e_{q}\right) \mathbb{R}\right\}$ решетки $A(Y)$. Поэтому $\alpha\left(\left(1-e_{p}\right) \mathbb{R}\right)=\left(1-e_{q}\right) \mathbb{R}$. По лемме $6 \alpha\left(M_{p}\right)=M_{q}$. Заметим, что подрешетка в $A(X)$, составленная из подалгебр на $X$, содержащихся в $M_{p}$, изоморфна решетке $A(X \backslash\{p\})$ при сужении функций из $M_{p}$ на хьюиттовское пространство $X \backslash\{p\}$. При этом подалгебре $\left(1-e_{p}\right) \mathbb{R}$ на $X$ отвечает подалгебра $\mathbb{R}$ на $X \backslash\{p\}$. Аналогично, подалгебры в $M_{q}$ образуют решетку, канонически изоморфную $A(Y \backslash\{q\})$. Следовательно, можно считать, что $\alpha$ осуществляет изоморфизм решетки $A(X \backslash\{p\})$ на решетку $A(Y \backslash\{q\})$, причем переводит $\mathbb{R}$ в $\mathbb{R}$. Согласно первому случаю подпространство $X \backslash\{p\}$ пространства $X$ гомеоморфно подпространству $Y \backslash\{q\}$ пространства $Y$. Очевидно, что тогда и $X \approx Y$.

4. О свойствах решетки $A(X)$. Будет показано, что решетка $A(X)$, как правило, немодулярна.

Пусть $X$ - произвольное $\mathbb{R}$-отделимое пространство. В зависимости от мощности $|X|$ пространства $X$ рассмотрим четыре случая.

1. При $|X|=1$ получаем цепь $A(X)=\{0, \mathbb{R}\}$.

2. Пусть $|X|=2$. Тогда $X=\{x, y\}$ - дискретное двоеточие. Получаем модулярную, но не дистрибутивную решетку $A(X)=\left\{0, \mathbb{R}, e_{x} \mathbb{R}, e_{y} \mathbb{R}, C(X)\right\}$.

3. Пусть $|X|=3$. Тогда $X=\{x, y, z\}$ - дискретное трехэлементное пространство. Решетка $A(X)$ немодулярна, ибо содержит немодулярную пятиэлементную подрешетку $\left\{0,\left(1-e_{z}\right) \mathbb{R},\left(1-e_{x}\right) \mathbb{R}, A_{y, z}, C(X)\right\}$. Имеем $|A(X)|=15$.

4. Пусть $|X| \geqslant 4$. Выберем в $X$ попарно различные точки $x_{1}, x_{2}, y_{1}, y_{2}$. Возьмем функцию $f \in C(X)$ так, чтобы $f\left(x_{1}\right)=f\left(x_{2}\right)=1$ и $f\left(y_{1}\right)=f\left(y_{2}\right)=0$. Рассмотрим подалгебры $[f]$ и $A=A_{x_{1}, y_{1}} \cap A_{x_{2}, y_{2}}$. Ясно, что

$$
A \cap[f]=\left\{r_{n} f^{n}+\cdots+r_{1} f: r_{i} \in \mathbb{R}, \sum_{i=1}^{n} r_{i}=0\right\}=A_{x_{1}, y_{1}} \cap[f] .
$$

Докажем равенство $A \vee[f]=C(X)$. Достаточно показать, что $A_{x_{1}, y_{1}} \subseteq A \vee[f]$. Возьмем $g \in A_{x_{1}, y_{1}}$. Так как $\mathbb{R} \subseteq A$, можно считать, что $g\left(x_{1}\right)=g\left(y_{1}\right)=0$. В силу 
$\mathbb{R}$-отделимости пространства $X$ существует функция $\varphi \in A$ такая, что $\varphi\left(x_{1}\right)=\varphi\left(y_{1}\right)=0$ и $\varphi\left(x_{2}\right)=\varphi\left(y_{2}\right)=g\left(x_{2}\right)-g\left(y_{2}\right)$. Для $h=g-f \varphi$ имеем

$$
h\left(x_{1}\right)=h\left(y_{1}\right)=0, \quad h\left(x_{2}\right)=g\left(x_{2}\right)-\left(g\left(x_{2}\right)-g\left(y_{2}\right)\right)=g\left(y_{2}\right)=h\left(y_{2}\right) .
$$

Значит, $h \in A$ и $g=f \varphi+h \in A \vee[f]$. В результате получаем в $A(X)$ немодулярную подрешетку $\left\{A \cap[f],[f], A, A_{x_{1}, y_{1}}, C(X)\right\}$.

Таким образом, доказана

ТЕОРема 3. Для любого $\mathbb{R}$-отделимого пространства $X$ имеют место утверждения:

а) решетка $A(X)$ модулярна $\Longleftrightarrow|X| \leqslant 2$;

б) решетка $A(X)$ дистрибутивна $\Longleftrightarrow|X|=1$.

ЗАмЕчАнИЕ. Вместо $\mathbb{R}$ можно брать различные топологические поля $E$, скажем, локально компактные поля (см. $[2, \S 2])$. Для них верны все результаты работы (с очевидными изменениями). Основной результат звучит так:

любое Е-компактное пространство $X$ определяется решеткой всех подалгебр Е-алгебры $C(X, E)$ всех непрерывных $E$-значных функиий на $X$.

В связи с изложенным естественно возникает следующая

ПроБЛЕмА. Дать описание всех максимальных подалгебр на хьюиттовских пространствах. В частности, не исчерпьваются ли подалгебрами вида $M_{x} u A_{x, y}$ все максимальные подалгебры в $C(X)$ для компактов $X$ ? Отметим, что по теореме Стоуна-Вейерштрасса среди замкнутых в равномерной топологии ( также в топологии поточечной сходимости) других максимальных подалгебр на компактах нет.

\section{СПИСОК ЦИТИРОВАННОЙ ЛИТЕРАТУРЫ}

[1] Gillman L., Jerison M. Rings of Continuous Functions. New York: Springer, 1976.

[2] Вечтомов Е. М. Вопросы определяемости топологических пространств алгебраическими системами непрерывных функций // Итоги науки и техн. Алгебра. Топология. Геометрия. Т. 28. М.: ВИНИТИ, 1990. С. 3-46.

[3] Биркгоф Г. Теория решеток. М.: Наука, 1984. 